\title{
A High Quality Steganographic Method Using Morphing
}

\author{
Anant M.Bagade* and Sanjay N.Talbar**
}

\begin{abstract}
A new morphed steganographic algorithm is proposed in this paper. Image security is a challenging problem these days. Steganography is a method of hiding secret data in cover media. The Least Significant Bit is a standard Steganographic method that has some limitations. The limitations are less capacity to hide data, poor stego image quality, and imperceptibility. The proposed algorithm focuses on these limitations. The morphing concept is being used for image steganography to overcome these limitations. The PSNR and standard deviation are considered as a measure to improve stego image quality and morphed image selection, respectively. The stego keys are generated during the morphed steganographic embedding and extracting process. Stego keys are used to embed and extract the secret image. The experimental results, which are based on hiding capacity and PSNR, are presented in this paper. Our research contributes towards creating an improved steganographic method using image morphing. The experimental result indicates that the proposed algorithm achieves an increase in hiding capacity, stego image quality, and imperceptibility. The experimental results were compared with state of the art steganographic methods.
\end{abstract}

Keywords-Morphed Steganography, Hiding Capacity, Imperceptibility, Stego Image Quality

\section{INTRODUCTION}

Information hiding has become an important in ubiquitous computing. Image morphing is a process of generating an animated sequence of images from one image to another. Morphing is derived from the word "metamorphosis." Morphing involves the image processing techniques of warping and cross dissolving. Morphing generates intermediate images, which are useful for hiding image data [1-4]. It is necessary that the presence of embedded information be kept secret for security purposes. Steganography is a technique of embedding information that is hard to detect. Steganography differs from cryptography in the sense that the presence of the message needs to remain secret, rather than focusing on the value of the message. Both steganography and cryptography have received a great deal of attention from law enforcement agencies and the media. Cryptography and steganography are the two important aspects of communication

※ The authors would like to thank the anonymous reviewers for their insightful comments and helpful suggestions to improve the quality of this paper.

Manuscript received March12, 2013; first revision August 13, 2013; second revision November 21, 2013; accepted January 27, 2014.

Corresponding Author: Anant M. Bagade (ambagade@pict.edu)

* Department of Information Technology, Pune Institute of Computer Technology, Pune-411043, Maharashtra, India (anantbagade@gmail.com, ambagade@pict.edu)

** Department of Electronics and Telecommunication SGGS Institute of Engineering and Technology, Nanded431606, Maharashtra, India (sntalbar@sggs.ac.in) 
security [5-7]. Images can serve as excellent carriers for hidden information. The common approach for steganography in images includes the Least Significant Bit (LSB) insertion method, and the frequency domain and spread spectrum techniques [8,9]. Each domain has its own advantages and disadvantages in terms of hiding capacity, quality, space complexity, and execution time. Two main factors that really affect the information hiding schemes are the embedding capacity and visual quality of a stego image. The digital steganography process has three basic components. First, there is the data that is to be hidden (secret data). Second, there is the secret data that is to be embedded (cover carrier) and third, there is the resulting stego file (stego carrier). Chan and Cheng [10] proposed a method for hiding data using a simple LSB substitution. An optimal pixel adjustment process is applied to a stego image to increase the embedding capacity and stego image quality. The PSNR obtained by this method was $51.1410 \mathrm{~dB}$. The concept of perceptual modeling is used to obtain a high quality embedding result in improved the steganographic method [11]. Chang et al. [12] proposed a method of finding the optimal LSB in image hiding by using a dynamic programming strategy to overcome the limitations of LSB substitution and the genetic algorithm. The hiding data always attempts to lower the distortion caused by doing so or to maximize the embedding payload with a given distortion level [13]. Human perceptibility has a property that it is sensitive to changes observed by the naked eye. Not all pixels in a cover image can tolerate equal amount of changes without causing noticeable distortion. Hence, to improve the stego image, several adaptive methods have been proposed in which the amount of bytes to be embedded in each pixel is variable. $\mathrm{Wu}$ and Tsa proposed a novel steganographic method that uses the difference value between two neighboring pixels to determine how many secret bits should be embedded [14].

Image morphing is basically used in the film and animation industry. Intermediate images are generated during the morphing process. The concept of morphing is useful in hiding information. A new information hiding scheme for image data using morphing is proposed in this paper. The proposed algorithm results in high embedding capacity, imperceptibility, and an increase in stego image quality. The main application area of the proposed work is in the field of military image security systems, medical diagnosis systems, security systems, and images used as evidence in court.

The remainder of this paper is organized as follows: in the next section, related work and the theoretical background of different steganographic methods with main contribution of morphed steganography is presented. The proposed theoretical background of morphing is discussed in Section 3. Morphed steganography is presented in Section 4. Our proposed morphed embedding and extraction algorithm is presented in Section 5. In Section 6 and Section 7 the experimental results and conclusion are presented, respectively.

\section{RELATED WORK}

Various research groups proposed a steganographic method [8-10,13-18] based on least significant bit modification. DCT steganography has advantages over the LSB based technique, in terms of achieving image quality at the cost of low data embedding capacity. DCT based steganography embeds the data in the least significant bits of the discrete cosine coefficient of the digital image. The comparative results stated in this paper show that the PSNR is high for DCT based steganography. A good quality image is indicated by a high PSNR ratio $[17,19]$. The 
chaos and genetic algorithms are used to achieve high embedding capacity and for preserving the quality of the image [18]. The tri-way pixel-value differencing approach achieves superior embedding capacity than the PVD method does. Neighboring pixel pairs are used to hide the information to achieve high embedding capacity. The result shows that the hiding capacity ratio of the proposed method and PVD approach is near 1.5 on average [14]. The correlation coefficient and its variation are used for distinguishing the existence of hidden data using LSB steganography [20]. Better visual quality is achieved using linear fusion by integrating information from different source images of a scene to form an image [21,22].The author used adaptive image filtering and adaptive image segmentation for bit replacement to get high capacity of secret data. Also, the comparison was done with the S-Tools algorithm, statistical attacks, and visual attacks $[23,24]$. The authors describe a high quality steganographic method with pixel-value differencing and a modulus function. The remainder of the two consecutive pixels is used to record the information of the secret data. It also solves the falling-off-boundary problem [25]. A comparative analysis of several image steganography methods, such as Mod10, DCT, PVD1, PVD2, stream of 1's and 0's, IP, and OPAP, were presented. The performance comparison, which is based on hiding capacity, PSNR, MSE, and time, is given [26,27]. The author has proposed a steganographic method for achieving high embedding capacity and security by using discrete wavelet transforms. A two level wavelet transform is applied as the cover and payload. The payload coefficients are encrypted and fused with wavelet coefficients of the cover image to generate stego coefficients, based on the embedding strength parameters of alpha and beta [28]. A universal approach to steganographic analysis using higher order statistics is described to show that these statistics are relatively consistent across a broad range of images, but are disturbed by the presence of hidden messages [29]. The author has presented a model for secure steganography based on embedding capacity. The proposed method determines the embedding capacity of an image regarding the efficient and well-known steganalysis methods and then clarifies the cover selection steganography based on embedding capacity [30]. The author proposed a new method for reliably estimating the length of a hidden message and for achieving reliability in the image, based on an analysis of adjacent pixel pairs. The results were predicted using the properties of the stego image [31]. High payload image steganography using two way block matching and a hop embedding scheme has been proposed for searching for the highest similarity between blocks for each block of the important image [32]. A novel multilayer information hiding scheme for the 3D polygon model has been proposed. It is based on a multilayered embedding scheme to hide secret messages in the vertices of a 3D polygon model [33]. The author carried out a performance study of the different steganographic algorithms and steganographic analysis. The image dataset is categorized with respect to size, quality, and texture to determine their potential impact on steganalysis performance [34,35]. Kondo and Zhao [3] proposed a steganographic method based on morphing for specific medical applications. LSB1 hides the secret data in each least significant bit of pixel of the cover image. Thus stego pixel of the cover image contains 7 MSB bits of cover and the last bit of a secret. Thus, 8 bits of cover can accommodate 1 bit of secret data. So, LSB1 has a hiding ratio of 1:8.Similarly LSB2, LSB3, and LSB4 will work on and their hiding ratios will be $2: 8,3: 8$, and $4: 8$, respectively [15]. The motivation of the proposed work is to identify new dimensions of morphing in steganography to improve the hiding capacity of secret data, stego image quality, and imperceptibility. 


\subsection{Main Contribution}

Any steganographic system is expected to work with a high embedding capacity, imperceptibility, and stego image quality. To achieve all of these requirements we have used morphing for hiding image data. Morphing is a process of generating the intermediate images from the source image to the destination image. Source image gradually fade towards the destination image by generating intermediate frames. Intermediate images contain some part of the source and some part of the destination image. This feature helps to hide any source or destination image between the selected intermediate images by using morphed steganography. The main contribution of this paper is summarized as follows: first, the proposed method generates intermediate images from the source to the destination using morphing. The stego keys are correctly identified and generated during the morphing process. The criterion for morphed image selection for hiding is identified by standard deviation and PSNR. Second, a new morphed steganographic method is proposed for hiding an image. The morphed embedding and extraction algorithm is designed by pixel value differencing cover image and secret image. Third, the proposed algorithm satisfies the property of imperceptibility, an increase in the hiding capacity of secret data, and stego image quality. Finally, this work compares the obtained results with some of the already existing methods.

\section{Proposed Work}

Steganographic methods are broadly classified in two categories. The first method is a universal steganographic technique, which is applicable to any technique and the second method is specific to the steganographic algorithm [26]. The proposed image data hiding algorithm is specific for an image morphing cross-dissolve technique. Our work is focus on increase in data hiding capacity, stego image quality, and imperceptibility. The proposed algorithm uses stego keys for morph generation. These stego keys are important to extract a secret image.The parameters used to define the proposed algorithm are given in Table1.The block schematic of the proposed method is given in Fig. 1.

Table 1. Parameters used to the define proposed algorithm

\begin{tabular}{c|c}
\hline Parameters & Functions \\
\hline $\mathrm{I}_{\mathrm{s}}$ & Secret source image \\
\hline $\mathrm{I}_{\mathrm{d}}$ & Destination cover image \\
\hline $\mathrm{I}_{\mathrm{Q}}$ & Set of morphed intermediate images \\
\hline $\mathrm{I}_{\mathrm{w}}$ & Stego data \\
\hline $\mathrm{K}$ & Stego keys \\
\hline $\mathrm{S}_{\mathrm{k}}$ & Stego image extraction \\
\hline $\mathrm{M}_{v^{\prime}}$ & De-morphing \\
\hline
\end{tabular}




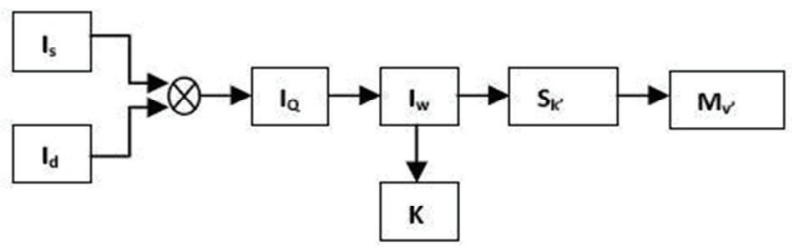

Fig. 1. Proposed system model

\subsection{Preprocessing}

The images are in a BMP grayscale format. They are 512x512 pixels in width and height and 72 pixels per inch resolution. BMP files use lossless compression, which is a scheme that allows to exactly reconstruct the original image.On the other hand, JPEGs use lossy compression, which means that the expanded image is almost the same as the original, but is not an exact duplicate. Lossless compression is much better suited to applications where the integrity of the original information must be maintained, such as for steganography. Two images are necessary for generating the morphed image set for further processing. The secret source image $I_{s}$ is defined as $\mathrm{I}_{\mathrm{s}}=\left\{\mathrm{s}_{1}, \mathrm{~s}_{2}, \ldots, \mathrm{s}_{\mathrm{n}}\right\}$ and $\mathrm{s}_{\mathrm{i}} \in \mathrm{A}$ such that $\forall \mathrm{i}=1,2, \ldots \mathrm{N}$, where $\mathrm{A}=\{0,1,2 \ldots, 255\}$. The destination cover image is defined as $\mathrm{I}_{\mathrm{d}}=\left\{\mathrm{d}_{1}, \mathrm{~d}_{2}, \ldots, \mathrm{d}_{\mathrm{n}}\right\}$ and $\mathrm{d}_{\mathrm{i}} \in \mathrm{A}$.

\subsection{Morphing}

Morphing is defined as the animated sequence of images from the source image to the destination image. The source image is gradually fed towards the destination image and vice

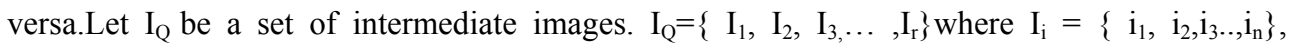
$\mathrm{ij} \in \mathrm{A}, \forall \mathrm{j}=1,2 \ldots \mathrm{N}$. Let $\mathrm{I}_{\mathrm{w}}$ be the stego data generated from steganography. $I_{\mathrm{w}}$ is defined as, $I_{w}=$ $\left\{\mathrm{w}_{1}, \mathrm{w}_{2}, \ldots, \mathrm{w}_{\mathrm{n}}\right\}, \quad \mathrm{w}_{\mathrm{i}} \in \mathrm{A}, \forall_{\mathrm{i}}=1,2 \ldots$. N.Let $\mathrm{K}$ be the stego keys generated from morphing process. $\mathrm{K}$ is defined as, $\mathrm{K}=\left\{\left\{\mathrm{S}_{\mathrm{k}}\right\},\left\{\mathrm{M}_{\mathrm{k}}\right\}\right\}$ where $\mathrm{S}_{\mathrm{k}}$ are the stego keys and are defined as $\mathrm{S}_{\mathrm{k}}=\left\{\mathrm{I}_{\mathrm{d}}, \mathrm{b}_{\mathrm{n}}\right\}$ where $I_{d}$ is the destination cover image and $b_{n}$ is the number of bits used in steganography. A maximum of 3 bits are used in the steganography. The length of $I_{d}$ is 256 bits and each bit is considered to be key. $M_{k}$ are the morphed keys and is defined as $M_{k}=\left\{I_{d}, N, r\right\}$, where $I_{d}$ is the destination cover image, $\mathrm{N}$ is the number of intermediate images to be generated, and $\mathrm{r}$ is the sequence number of intermediate images selected. The threshold value of $\mathrm{N}$ is decided by calculating the standard deviation between different intermediate images and the value of $\mathrm{N}$ is 225(see Fig.4). Intermediate morphed images are generated using $M_{v}$ and is defined as, $M_{v}=\left\{I_{s}\right.$, $I_{d}, M_{k}$, which generates $I_{Q}, M_{v}$ takes $I_{s}, I_{d}$, and $M_{k}$ as input and generates the $N$ intermediate images, where $N \in M_{k}$. Every pixel in intermediate image $I_{j}$ is generated using the following equation:

$$
\begin{aligned}
& I_{j}=(1-j / N) I_{s}+(j / N) I_{d} \\
& \text { Thus, } I_{0}=I_{s} \text { and } I_{N}=I_{d} .
\end{aligned}
$$

\subsection{Morphed Image Selection}

One intermediate image is necessary for morphed steganography out of $\mathrm{N}$ intermediate images generated during the morphing process . The selected morphed image is embedded into a 
cover destination image. One image out of $\mathrm{N}$ intermediate images is selected using the following function:

$$
\mathrm{F}: \mathrm{I}_{\mathrm{Q}} \rightarrow \mathrm{I}_{\mathrm{r}}
$$

Function F is used to select the suitable image for steganography. The selection is based on the criteria listed below.

Minimize Standard Deviation is calculated as, $\sigma\left(\mathrm{I}_{\mathrm{r}} \mathrm{I}_{\mathrm{d}}\right)$ subject to PSNR $\mathrm{I}_{\mathrm{w}}(\mathrm{r})>36 \mathrm{~dB}$ where $\mathrm{I}_{\mathrm{w}}(\mathrm{r})$ is the stego data produced with the help of $\mathrm{I}_{\mathrm{r} .}$ The experimental results displayed in Table 4 indicate that the PSNR value of LSB-4 is $33.889 \mathrm{~dB}$. The LSB-4 changes 4 bits out of 8 per pixel for steganography. By considering the aspect of the human visual system the change is $50 \%$ and is recognized by normal human eyes, which does not satisfy the property of imperceptibility. The same experiment was carried out for LSB-3, as shown in Table 4 and the PSNR value is $37.856 \mathrm{~dB}$. The hidden data is 3 bits and is not recognized by human eyes. The mean value of the PSNR of LSB-4 and LSB-3 is 36dB and hence, is considered as a threshold for measuring quality.Standard Deviation is calculated using the following formula:

$$
\sigma\left(I_{r}, I_{d}\right)=\sqrt{\frac{1}{n} \sum_{1}^{n}\left(I_{r}(i, j)-I_{d}(i, j)\right)^{2}}
$$

The Mean Squared Error (MSE) measures the average squares of the error. The error is the amount by which the value implied by the estimator differs from the quantity to be estimated.The Peak Signal-to-Noise Ratio (PSNR) is calculated using the following formula:

$$
P S N R=10 \log _{10} \frac{255^{2}}{M S E}
$$

Where $M S E=\frac{1}{n} \sum_{i=1}^{n}\left(X_{i}-X_{i}^{\prime}\right)^{2}$

\section{Morphed Steganography}

Morphed steganography is defined by using the function SK and is defined as SK: $\left(I_{s}, I_{d}, S_{k}\right)$ $\rightarrow I_{w}$. The function SK accepts $I_{s}, I_{d}, S_{k}$ as input and using morphed steganography it generates stego data $\mathrm{I}_{\mathrm{w}}$. The morphed steganography has uses the following steps:

Let $D^{\prime}$ be the difference set

Step 1. D' $=I_{r}-I_{d}$

Step 2. $\mathrm{D}=\mathrm{D}^{\prime}+3$

Step 3. $\mathrm{I}_{\mathrm{w}}=\mathrm{I}_{\mathrm{d}} \otimes \mathrm{D}$

Where $\otimes$ is an operator that replaces the last $b_{n}$ bit of $I_{d}$ with D.Step 1 calculates the difference set of pixels from one selected image $I_{r}$ and the cover destination image $I_{d}$. The number 3 is added to each difference value to make the pixel a positive value. The value of difference set D' is in the range of 0 to 2 . This means each pixel difference is in the range of 0 to 2 . A maximum of two bits are used to store this pixel difference. The stego image $I_{w}$ is generated by a replacement of the difference bit value with the last $b_{n}$ bit of each pixel of $I_{d}$ with $D$. 


\subsection{Secret Extraction from StegoData}

Let $\mathrm{S}_{\mathrm{k}}$ : $\left(\mathrm{I}_{\mathrm{w}}{ }^{\prime}, \mathrm{S}_{\mathrm{k}}\right) \rightarrow \mathrm{I}_{\mathrm{r}}{ }^{\prime}$ be a function that takes $\mathrm{I}_{\mathrm{w}}{ }^{\prime}, \mathrm{S}_{\mathrm{k}}$ as input and with the help of $\mathrm{S}_{\mathrm{k}}$ it extracts

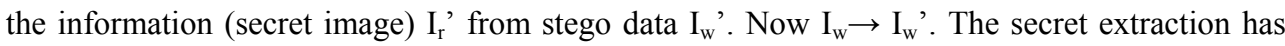
uses the following steps:

Step 1. $\mathrm{D}=\mathrm{S}_{\mathrm{k}},\left(\mathrm{I}_{\mathrm{w}}{ }^{\prime}\right)$

Step 2. $S_{k}$, returns the last $b_{n}$ bits of each pixel from $I_{w}$ '

Step 3. D'= D-3

Step 4. $\mathrm{I}_{\mathrm{r}}{ }^{\prime}=\mathrm{I}_{\mathrm{d}}+\mathrm{D}^{\prime}$

The difference set $D$ contains the last $b_{n}$ bit of each pixel from $I_{w}{ }^{\prime}$. The number 3 is subtracted from $\mathrm{D}$ to get the original pixel value. The secret stego data is extracted after adding $\mathrm{D}$ ' with $\mathrm{I}_{\mathrm{d}}$.

\subsection{De-Morphing}

De-Morphing is the reverse step of morphing. It is used to get the original secret image. Let $M_{v}{ }^{\prime}:\left(I_{r}{ }^{\prime}, M_{k}\right) \rightarrow I_{s}{ }^{\prime}$ be a function that takes $I_{r}{ }^{\prime}, M_{k}$ as input and with the help of $M_{k}$ the image $I_{r}{ }^{\prime}$ is De-Morphed to generate the secret image $I_{s}{ }^{\prime}$. The function $M_{v}{ }^{\prime}$ generates the $I_{s}{ }^{\prime}$ with the following equation:

$$
I_{s}{ }^{\prime}=\frac{I_{r}{ }^{\prime}-\frac{r}{N} I_{d}}{1-\frac{r}{N}}
$$

\section{MoRphed Embedding And Extraction Algorithm}

Table 2. Pseudo code for the Morphed Embedding Algorithm

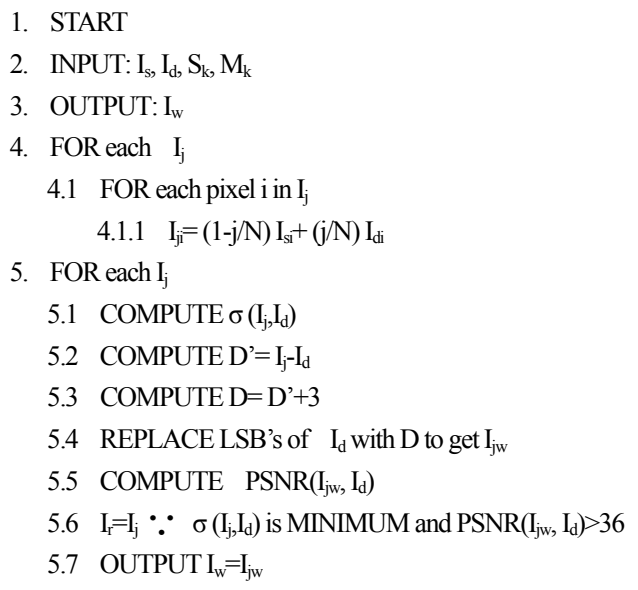

2. INPUT: $I_{s}, I_{d}, S_{k}, M_{k}$

3. OUTPUT: $I_{w}$

4. FOR each $\mathrm{I}_{\mathrm{j}}$

4.1 FOR each pixel i in $\mathrm{I}_{\mathrm{j}}$

4.1.1 $\mathrm{I}_{\mathrm{j} i}=(1-\mathrm{j} / \mathrm{N}) \mathrm{I}_{\mathrm{s}}+(\mathrm{j} / \mathrm{N}) \mathrm{I}_{\mathrm{di}}$

5. FOR each $\mathrm{I}_{\mathrm{j}}$

5.1 COMPUTE $\sigma\left(\mathrm{I}_{\mathrm{j}} \mathrm{I}_{\mathrm{d}}\right)$

5.2 COMPUTE $\mathrm{D}^{\prime}=\mathrm{I}_{\mathrm{j}}-\mathrm{I}_{\mathrm{d}}$

5.3 COMPUTE $\mathrm{D}=\mathrm{D}^{\prime}+3$

5.4 REPLACE LSB's of $\mathrm{I}_{\mathrm{d}}$ with $\mathrm{D}$ to get $\mathrm{I}_{\mathrm{jw}}$

5.5 COMPUTE PSNR $\left(\mathrm{I}_{\mathrm{j},}, \mathrm{I}_{\mathrm{d}}\right)$

$5.6 \mathrm{I}_{\mathrm{r}}=\mathrm{I}_{\mathrm{j}} \because \sigma\left(\mathrm{I}_{\mathrm{j} j} \mathrm{I}_{\mathrm{d}}\right)$ is MINIMUM and PSNR $\left(\mathrm{I}_{\mathrm{j},}, \mathrm{I}_{\mathrm{d}}\right)>36$

5.7 OUTPUT $\mathrm{I}_{\mathrm{w}}=\mathrm{I}_{\mathrm{jw}}$

6. END 
Table 3. Pseudo code for the Morphed Extraction Algorithm
1. START
2. INPUT: $I_{W}$
3. OUTPUT: $I_{S}$
4. ACCEPT LSB's of $I_{w}$ in $D$
5. COMPUTE $\mathrm{D}^{\prime}=\mathrm{D}-3$
6. COMPUTEI $=\mathrm{I}_{\mathrm{d}}+\mathrm{D}^{\prime}$
7. FOR each pixel $\mathrm{i}$ in $\mathrm{I}_{\mathrm{s}}$

$$
I_{s}(i)=I_{s}{ }^{\prime}=\frac{I_{r}{ }^{\prime}-\frac{r}{N} I_{d}}{1-\frac{r}{N}}
$$
8. END

The pseudo code for morphed embedding and extraction algorithm is given in Table 2 and Table 3, respectively. The purpose of using the morphed embedding algorithm is to hide the secret image in the cover image by selecting the appropriate morphed image. The condition for morphed image selection is stated in the algorithm. The proposed embedding algorithm hides the difference value pixel between the morphed and destination images. Algorithm complexity is reduced because of the pixel difference value being stored. Morphed extraction is the reverse process of morphed embedding. The original secret image is extracted from the stego data by using the stego keys. A detailed algorithm explanation is given in Sections 3 and 4 . The advantages of these proposed algorithms are an increase in hiding capacity of secret data, improved stego image quality, and imperceptibility.

\subsection{Algorithm Analysis}

The time complexity of the stated algorithm in Table 2 and Table 3 is $O\left(n^{2}\right)$ and $O(n)$, respectively,for different input size images in worst case. The minimum number of steps required for the stated algorithm is $\mathrm{n}^{2}$ and $\mathrm{n}$, respectively. So, the complexity of the algorithm is $\Omega\left(\mathrm{n}^{2}\right)$ and $\Omega(\mathrm{n})$, respectively. The number of steps required, or the upper bound and lower bound, is the same for the proposed algorithm. As such, the complexity for the stated algorithm is $\Phi\left(\mathrm{n}^{2}\right)$ and $\Phi(\mathrm{n})$, respectively. The proposed algorithm is asymptotically tight.

\section{RESULTS}

In this section, we present the experimental results that came from using our proposed method. We implemented our algorithm in Java language and the running environment was an AMD Phenom[tm]IIx4955 processor,3.21 GHz,3.25 GB of RAM with a Windows XP operating system. The algorithm was processed on 512 x 512 pixels with 8 bits/pixels BMP gray scale images. A standard image set of 1,000 images were considered as the experimental images for this algorithm [36].The output of the morphed steganographic algorithm is shown in Fig.5. 
Table 4. Comparison of steganographic algorithms with average hiding capacity, average PSNR, Time, and PSNR Gain

\begin{tabular}{c|c|c|c|c}
\hline $\begin{array}{c}\text { LSB } \\
\text { Algorithms }\end{array}$ & $\begin{array}{c}\text { Average Hiding } \\
\text { Capacity } \\
\text { (in bits) }\end{array}$ & Average PSNR(dB) & $\begin{array}{c}\text { Time } \\
\text { (in seconds) }\end{array}$ & $\begin{array}{c}\text { PSNR } \\
\text { Gain w.r.t. our } \\
\text { method(dB) }\end{array}$ \\
\hline LSB-1 & 32,400 & 51.117 & 1.68 & 0.003 \\
\hline LSB-2 & 65,536 & 44.398 & 1.86 & 6.704 \\
\hline LSB-3 & 97,969 & 37.856 & 1.97 & 13.26 \\
\hline LSB-4 & 131,044 & 33.889 & 2.16 & 17.23 \\
\hline SLSB & 131,072 & 44.016 & 2.14 & 7.104 \\
\hline OLSB & 65,536 & 44.365 & 1.16 & 6.755 \\
\hline OPAP & 78,363 & 46.157 & 1.37 & 4.963 \\
\hline Chung-Ming Wang, et al. & 51,660 & 43.641 & 1.13 & 7.479 \\
\hline Ko-Chin Chang, et al. & 77,543 & 37.505 & 1.36 & 13.61 \\
\hline Our method & 262,144 & 51.120 & 0.97 & - \\
\hline
\end{tabular}

Table 5.Proposed morphed steganography algorithm results

\begin{tabular}{|c|c|c|c|}
\hline & & \multicolumn{2}{|c|}{ Proposed Method } \\
\hline $\begin{array}{l}\text { Cover } \\
\text { Image } \\
\text { Category } \\
(512 \times 512) \\
\end{array}$ & $\begin{array}{l}\text { Image } \\
\text { Name }\end{array}$ & $\begin{array}{l}\text { Morphed Image Hid- } \\
\text { ing Capacity } \\
\text { (in bytes) }\end{array}$ & $\operatorname{PSNR}(\mathrm{dB})$ \\
\hline \multirow{4}{*}{ Face } & Lena & 262,144 & 50.9987 \\
\hline & Baboon & 262,144 & 51.0149 \\
\hline & Magician & 262,144 & 51.2887 \\
\hline & Lion & 262,144 & 51.1475 \\
\hline \multirow{5}{*}{ Abstract } & Grass & 262,144 & 51.1403 \\
\hline & Bark & 262,144 & 51.2992 \\
\hline & Straw & 262,144 & 51.0614 \\
\hline & Woolen & 262,144 & 51.2969 \\
\hline & Pigskin & 262,144 & 51.1323 \\
\hline \multirow{5}{*}{ Object } & Tank & 262,144 & 51.0300 \\
\hline & Jet & 262,144 & 51.0300 \\
\hline & Boat & 262,144 & 51.1008 \\
\hline & Bridge & 262,144 & 51.0788 \\
\hline & Car & 262,144 & 51.0722 \\
\hline
\end{tabular}




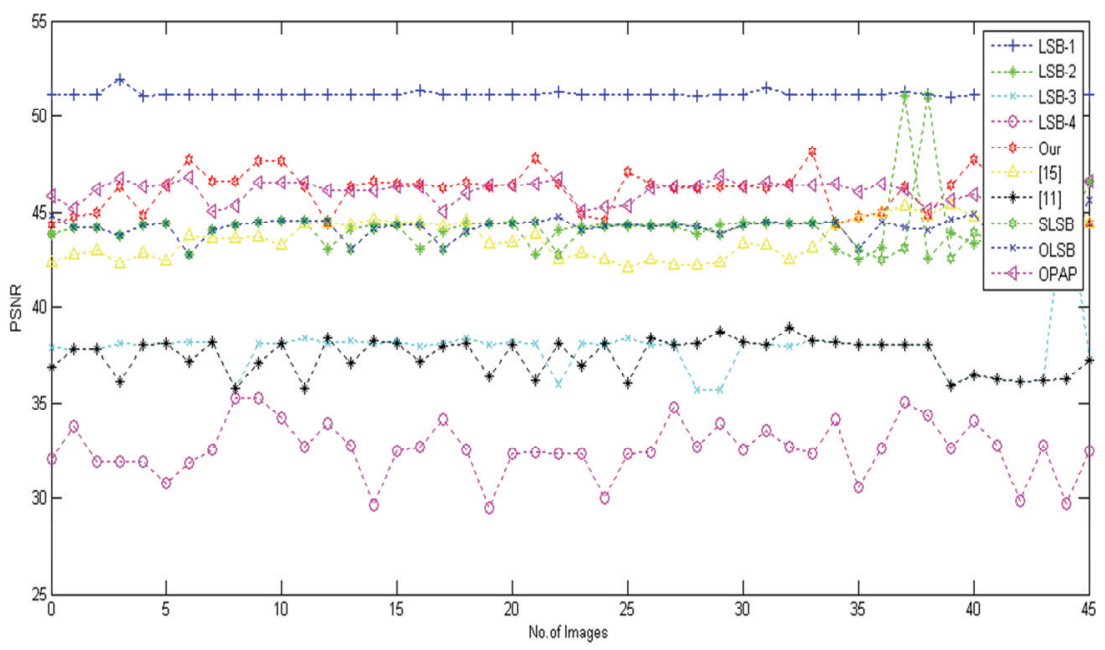

Fig. 2. Comparative PSNR performance

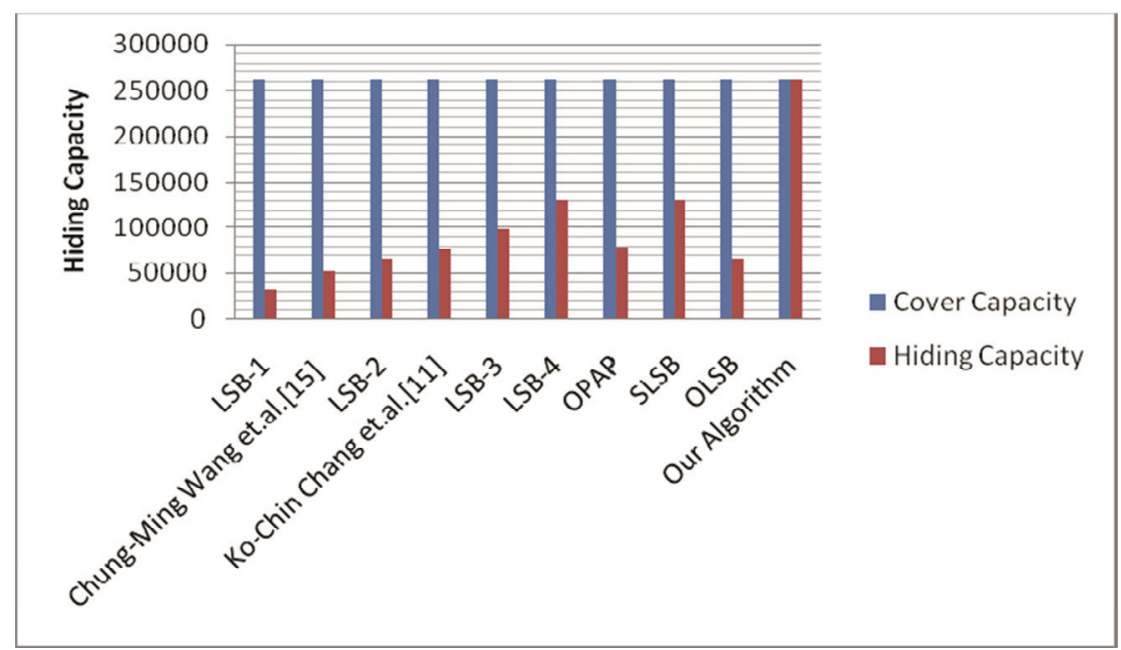

Fig. 3. Comparative hiding capacity 


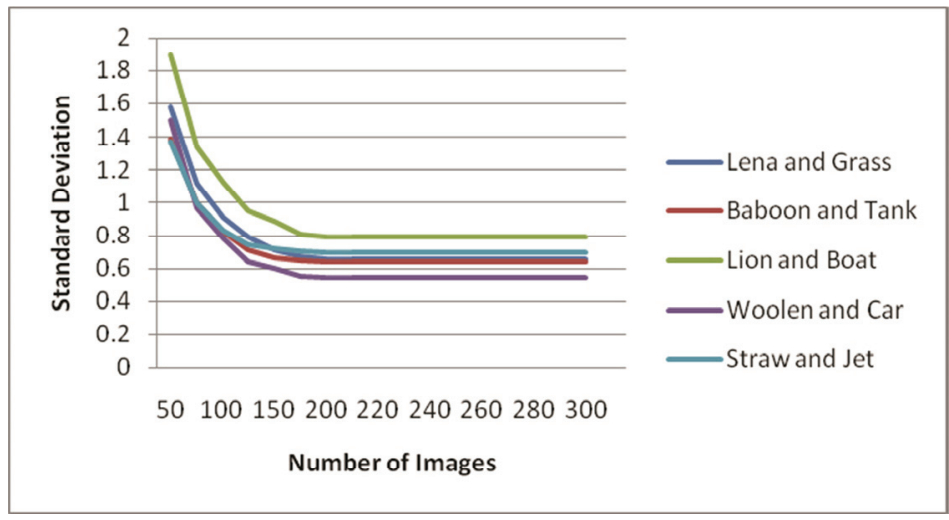

Fig. 4. Number of images vs. Standard Deviation

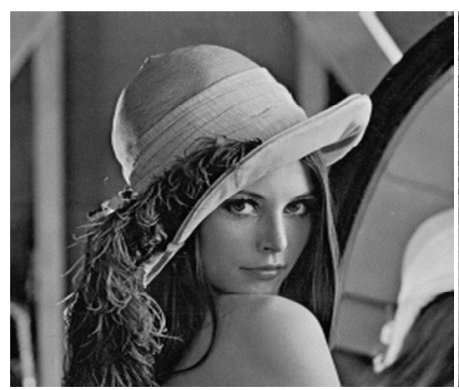

$\mathrm{I}_{\mathrm{s}}$

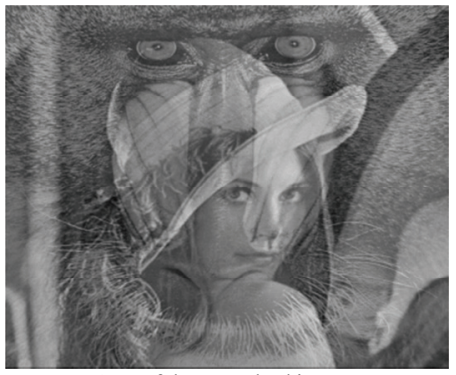

One of the morphed images

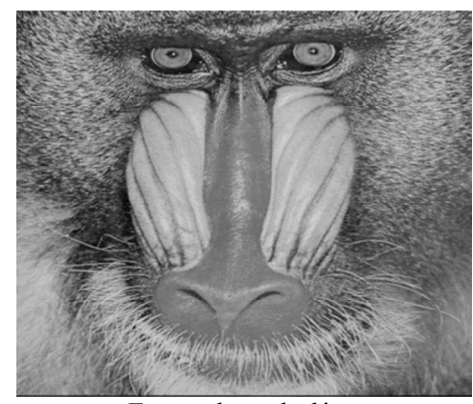

Extracted morphed image

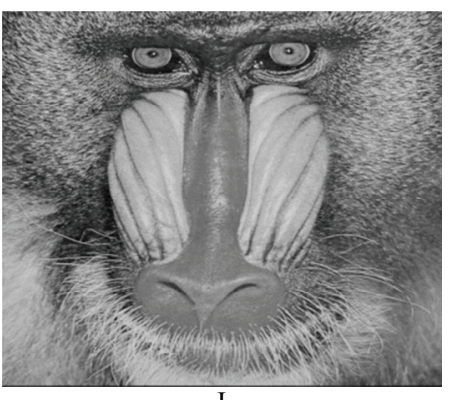

$\mathrm{I}_{\mathrm{d}}$

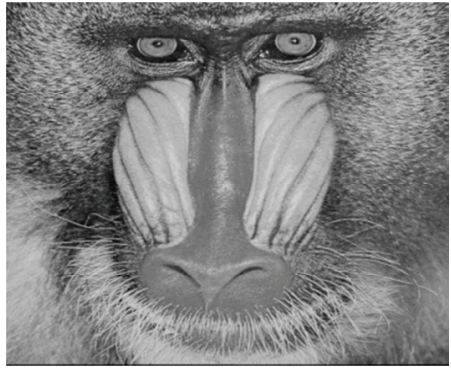

$\mathrm{I}_{\mathrm{w}}$

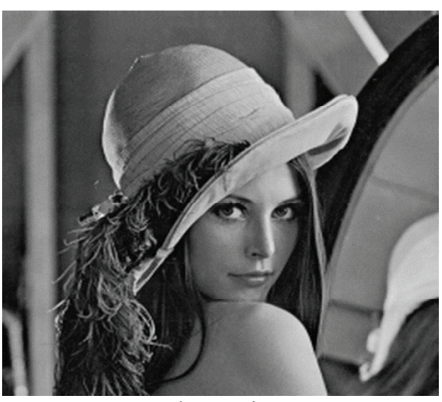

Extracted secret image

Fig. 5. Results of the morphed steganographic algorithm 
The PSNR and hiding capacity of secret data are being used as a measure to evaluate the performance of standard algorithms and our proposed algorithm. The results were presented in Table 4 and Table 5, respectively. The cover capacity, average hiding capacity, average PSNR, and the PSNR gain with our method and running time for steganographic algorithms are summarized in Table 4. Table 4 indicates that the hiding capacity of secret data increases with respect to other standard algorithms, such as LSB-1 to LSB-4 [15], SLSB [37], OLSB [37], OPAP [37], Wang et al. [25], and Chang et al. [14], with decreases in the PSNR. The PSNR indicates the imperceptibility of the secret image and cover image. The PSNR decreases, which indicates that the hidden image can be identified by normal eyes and it violates the imperceptibly property. To bridge the gap between these two measures, our proposed algorithm gives satisfactory results. Table 4 also indicates the PSNR gain with other algorithms. All of the algorithms mentioned in Table 4 were experimented on and the running time was calculated for each algorithm. The running time of our proposed algorithm was less than the other algorithms. This was because of the small pixel difference between the selected morphed image and the secret image. Less computational time was required because only the difference pixel values needed to be modified. Table 5 shows the results of the morphed image hiding capacity and PSNR of the proposed algorithm on different image sets. The PSNR value is not corrupted in the image categories. Fig. 2 shows the comparative PSNR performance of a standard algorithm and our proposed algorithm. The PSNR of the proposed algorithm varies from $46 \mathrm{~dB}$ to $51 \mathrm{~dB}$ and satisfies the property of imperceptibility. The normal condition for imperceptibility is PSNR should be greater than 36dB.The proposed algorithm's PSNR is higher than this value. The PSNR of the proposed algorithms is higher than other algorithms, except for LSB-1. It is inferior to LSB-1, but the hiding capacity of the proposed algorithm is higher than LSB-1.The proposed algorithm's PSNR is increased by $15.72 \%$, as compared to standard algorithms. Fig. 3 shows the comparative hiding capacity of standard algorithms and the proposed algorithm. The capacity to hide secret data increased because of storing the difference bits of the selected morphed image and cover image. Fig. 4 shows the threshold value for generating the number of morphed images and it is 225 . The standard deviation for intermediate images from source to destination remains the same after obtaining this threshold value. The stego image is generated by selecting the $\mathrm{N}-1^{\text {th }}$ image and destination image. The pixel difference between these two images ranges from 0 to 2 pixels. These pixel difference values are used for steganography. The proposed algorithm increases the hiding capacity of secret data, improves the quality of the stego image, and satisfies the property of imperceptibility.

\section{CONCLUSION}

This paper introduced a new morphed steganographic algorithm that is capable of increasing the hiding capacity of secret data, and improving image quality and imperceptibility. The hiding capacity of secret data increases due to consideration of only pixel difference between the selected morphed image and the destination image.There is a gain of the PSNR with standard algorithms, as compared to the proposed algorithm. The high value of the PSNR results in the good quality of a stego image and proves the imperceptibility property of steganography. Our proposed algorithm's PSNR is increased by $15.72 \%$ in comparison to existing algorithms that we have mentioned. The hiding capacity of secret data is also increased in comparison to 
existing algorithms. Owing its ability to an increase in hiding capacity of secret data and imperceptibility, it can be applied to any image data security applications. The future direction will be to find out the mechanism for transmitting the stego keys securely over the Internet. The PSNR between the stego image and cover image is high, so there is a scope for embedding more secret data in the stego image. The proposed algorithm can be extended for multilevel embedding, which will give an even higher embedding capacity.

\section{REFERENCES}

[1] T. Kanai, H. Suzuki, and F. Kimura, "Metamorphosis of arbitrary triangular meshes," IEEE Computer Graphics and Applications, vol. 20, no. 2, pp. 62-75, 2000.

[2] H. Nakamura and Z. Qiangfu, "Information hiding based on image morphing," in Proceedings of the 22nd International Conference on Advanced Information Networking and Applications, Okinawa, Japan, 2008, pp. 1585-1590.

[3] S. Kondo and Q. Zhao, "A novel steganographic technique based on image morphing," in Ubiquitous Intelligence and Computing: Proceedings of the Third International Conference, Wuhan, China, September 3-6, 2006 (Lecture Notes in Computer Science Vol. 4159), J. Ma, H. Jin, L. Yang, and J. P. Tsai, Eds. Heidelberg, Germany: Springer Berlin, 2006, pp. 806-815.

[4] A. M. Bagade and S. N. Talbar, "Image morphing concept for secure transmission of image data contents over internet," Journal of Computer Science, vol. 6, no. 9, pp. 987-992, 2010.

[5] W. Ying and P. Moulin, "Perfectly secure steganography: capacity, error exponents, and code constructions," IEEE Transactions on Information Theory, vol. 54, no. 6, pp. 2706-2722, 2008.

[6] S. Narayana and G. Prasad, "Two new approaches for secured image steganography using cryptographic techniques and type conversions," Signal \& Image Processing, vol. 1, no. 2, pp. 60-73, 2010.

[7] P. A. Regalia, "Cryptographic secrecy of steganographic matrix embedding," IEEE Transactions on Information Forensics and Security, vol. 3, no. 4, pp. 786-791, 2008.

[8] M. Mohamed, F. Al-Afari, and M. Bamatraf, "Data hiding by LSB substitution using genetic optimal key-permutation," International Arab Journal of e-Technology, vol. 2, no. 1, pp. 11-17, 2011.

[9] X. Liao, Q. Y. Wen, and J. Zhang, "A steganographic method for digital images with four-pixel differencing and modified LSB substitution," Journal of Visual Communication and Image Representation, vol. 22, no. 1, pp. 1-8, 2011.

[10] C. K. Chan and L. M. Cheng, "Hiding data in images by simple LSB substitution," Pattern Recognition, vol. 37, no. 3, pp. 469-474, 2004.

[11] R. Z. Wang, C. F. Lin, and J. C. Lin, "Image hiding by optimal LSB substitution and genetic algorithm," Pattern Recognition, vol. 34, no. 3, pp. 671-683, 2001.

[12] C. C. Chang, J. Y. Hsiao, and C. S. Chan, "Finding optimal least-significant-bit substitution in image hiding by dynamic programming strategy," Pattern Recognition, vol. 36, no. 7, pp. 1583-1595, 2003.

[13] Z. Xinpeng, "Efficient data hiding with plus-minus one or two," IEEE Signal Processing Letters, vol. 17, no. 7, pp. 635-638, 2010.

[14] K. C. Chang, C. P. Chang, P. S. Huang, and T. M. Tua, "A novel image steganographic method using tri-way pixel-value differencing," Journal of Multimedia, vol. 3, no. 2, pp. 37-44, 2008.

[15] G. Swain and S. Lenka, "LSB array based image steganography technique by exploring the four least significant bits," in Global Trends in Information Systems and Software Applications (Communications in Computer and Information Science Vol. 270), P. V. Krishna, M. R. Babu, and E. Ariwa, Eds. Heidelberg, Germany: Springer Berlin, 2012, pp. 479-488.

[16] C. H. Yang, "Inverted pattern approach to improve image quality of information hiding by LSB substitution," Pattern Recognition, vol. 41, no. 8, pp. 2674-2683, 2008.

[17] E. Walia, P. Jain, and Navdeep, "An analysis of LSB \& DCT based steganography," Global Journal of Computer Science and Technology, vol. 10, no. 1, pp. 4-8, 2010.

[18] L. Yu, Y. Zhao, R. Ni, and T. Li, "Improved adaptive LSB steganography based on chaos and genetic 
algorithm," EURASIP Journal on Advances in Signal Processing, vol. 2010, no. 1, pp. 876946, 2010.

[19] H. B. Kekre, A. Athawale, and P. N. Halarnkar, "Increased capacity of information hiding in LSB's method for text and image," World Academy of Science, Engineering and Technology, vol. 2, no. 5, pp. 657-660, 2008.

[20] A. Yadollahpour and H. M. Naimi, "Attack on LSB steganography in color and grayscale images. using autocorrelation coefficients," European Journal of Scientific Research, vol. 31, no. 2, pp. 172 $183,2009$.

[21] C. H. Hsieh and Q. Zhao, "Image enhancement and image hiding based on linear image fusion," in Image Fusion [Online], O. Ukimura, Ed. Intech, 2011, Chapter 2. Available: http://dx.doi.org/10.5772/14331

[22] M. Kharrazi, H. Sencar, and N. Memon, "Improving steganalysis by fusion techniques: a case study with image steganography," in Transactions on Data Hiding and Multimedia Security I (Lecture Notes in Computer Science Vol. 4300), Y. Shi, Ed. Heidelberg, Germany: Springer Berlin, 2006, pp. 123-137.

[23] N. N. EL-Emam, "Hiding a large amount of data with high security using steganography algorithm," Journal of Computer Science, vol. 3, no. 4, pp. 223-232, 2007.

[24] A. Westfeld and A. Pfitzmann, "Attacks on steganographic systems," in Information Hiding: Third International Workshop, IH'99, Dresden, Germany, September 29-October 1, 1999 Proceedings (Lecture Notes in Computer Science Vol. 1768), A. Pfitzmann, Ed. Heidelberg, Germany: Springer Berlin, 2000, pp. 61-76.

[25] C. M. Wang, N. I. Wu, C. S. Tsai, and M. S. Hwang, "A high quality steganographic method with pixel-value differencing and modulus function," Journal of Systems and Software, vol. 81, no. 1, pp. 150-158, 2008.

[26] A. Nissar and A. H. Mir, "Classification of steganalysis techniques: a study," Digital Signal Processing, vol. 20, no. 6, pp. 1758-1770, 2010.

[27] R. Amirtharajan, R. Akila, and P. Deepikachowdavarapu, "A comparative analysis of image steganography," International Journal of Computer Applications, vol. 2, no. 3, pp. 41-47, 2010.

[28] H. S. Manjunatha Reddy and K. B. Raja, "High capacity and security steganography using Discrete Wavelet Transform," International Journal of Computer Science and Security, vol. 3, no. 6, pp. 462472, 2010.

[29] L. Siwei and H. Farid, "Steganalysis using higher-order image statistics," IEEE Transactions on Information Forensics and Security, vol. 1, no. 1, pp. 111-119, 2006.

[30] H. Sajedi and M. Jamzad, "Secure steganography based on embedding capacity," International Journal of Information Security, vol. 8, no. 6, pp. 433-445, 2009.

[31] R. Shreelekshmi, M. Wilscy, and C. E. V. Madhavan, "Cover image preprocessing for more reliable LSB replacement steganography," in Proceedings of the International Conference on Signal Acquisition and Processing, Bangalore, India, 2010, pp. 153-156.

[32] R. Z. Wang and Y. S. Chen, "High-payload image steganography using two-way block matching," IEEE Signal Processing Letters, vol. 13, no. 3, pp. 161-164, 2006.

[33] M. W. Chao, C. H. Lin, C. W. Yu, and T. Y. Lee, "A high capacity 3D steganography algorithm," IEEE Transactions on Visualization and Computer Graphics, vol. 15, no. 2, pp. 274-84, 2009.

[34] I. Avcibas, N. Memon, and B. Sankur, "Steganalysis using image quality metrics," IEEE Transactions on Image Processing, vol. 12, no. 2, pp. 221-229, 2003.

[35] M. Kharrazi, H. T. Sencar, and N. D. Memon, "Performance study of common image steganography and steganalysis techniques," Journal of Electronic Imaging, vol. 15, no. 4, pp. 041104, 2006.

[36] The USC-SIPI Image Database [Online]. Available: http://sipi.usc.edu/database/

[37] S. F. Mare, M. Vladutiu, and L. Prodan, "Decreasing change impact using smart LSB pixel mapping and data rearrangement," in Proceedings of the IEEE 11th International Conference on Computer and Information Technology, Pafos, Cyprus, 2011, pp. 269-276. 


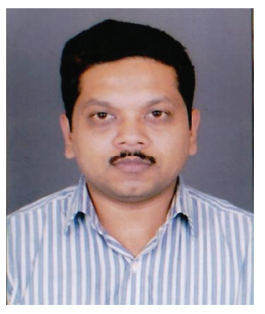

\section{Anant M.Bagade}

He received Bachelor of Engineering (Computer Science \& Engineering) from Amravati University, Maharashtra, India, in year 1999 and received Masters in Computer Engineering from Pune University, Maharashtra, India in year 2005. He is currently a Research Scholar at SGGS Institute of Engineering and Technology affiliated to Swami Ramanand Teerth Marathwada University Nanded, Maharashtra, India. He is currently working as a Associate Professor with Department of Information Technology, Pune Institute of Computer Technology, Pune, India. He received a "Best Paper Award" in year 2005 by IEEE. He has published research papers in reputed international journals. His research interest includes Image Processing, Data Security, and Brain Computer Interface etc.

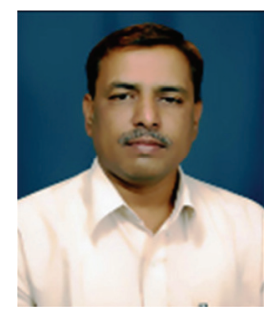

\section{Dr. Sanjay N.Talbar}

$\mathrm{He}$ is a Professor in Electronics and Telecommunications Engineering department at SGGS Institute of Engineering and Technology Nanded, Maharashtra, India. He received $B E$ and ME degrees in Electronics Engineering from the Marathwada University, Aurangabad, India, in1985 and in 1990,respectively and Ph.D from S.R.T. Marathwada University, Nanded in May 2000.He worked at various levels in Academic, Research and professional organizations. He received the "Young Scientist Award" by URSI, Italy in 2003. He has Collaborative research program at Cardiff University Wales, UK. He is an author of several books. He published about 45 research papers in reputed journal and about 110 papers in conferences. He guided more than 60 students for Dissertation at M.Tech. and completed 11 students for PhD thesis. Dr. Talbar's area of interest includes Image processing, Multimedia Computing Systems, Real Time Embedded system and Design, Pattern Recognition and Machine Intelligence, Biometrics and Texture Analysis etc. He is a member of several professional societies like ISTE, IETE. 